\title{
ON THE FORMATION OF SHOCKS TO THE COMPRESSIBLE EULER EQUATIONS *
}

\author{
DONGHO CHAE $\dagger$ AND SEUNG-YEAL HA $\ddagger$
}

\begin{abstract}
We present an explicit upper-bound for the life-span of $C^{3}$-smooth solutions to the multi-dimensional compressible Euler equations with a certain class of initial data containing compression vacuum states. We also show that the divergence of a fluid velocity will blow up along the particle trajectories issued from the compression vacuum states, which represent a shock formation at the vacuum states.
\end{abstract}

Key words. Compressible Euler equations, blow-up, shock wave, particle trajectory, vacuum boundary.

AMS subject classifications. 35Q35, 35B30.

1. Introduction The formation of singularities such as shocks, vacuum states, and the loss of regularity in fluid variables across the fluid-vacuum interface are generic nonlinear phenomena often found in compressible fluids, and the mathematical study of singularity formation and its dynamics has been addressed in previous literature $[3,4,7,8,9,10,13,11,14,15,19,20,22,23]$. However, detailed understanding on the formation and dynamics of singularities is mostly confined to the one-dimensional case. In this paper, we are interested in the formation of shocks at the compression vacuum states present in multi-dimensional inviscid, compressible fluids. In this case, the motion of an inviscid, compressible fluids is governed by the compressible Euler equations with a suitable equation of state and initial data:

$$
\begin{aligned}
& \partial_{t} \rho+\operatorname{div}(\rho v)=0, \quad x \in \mathbb{R}^{d}, \quad t>0, \\
& \partial_{t}(\rho v)+\operatorname{div}(\rho v \otimes v)=-\nabla p, \\
& \partial_{t} S+(v \cdot \nabla) S=0, \\
& p=\kappa e^{S} \rho^{\gamma}, \quad(\kappa>0, \quad \gamma>1),
\end{aligned}
$$

subject to initial data

$$
(\rho, v, S)(x, 0)=\left(\rho_{0}, v_{0}, S_{0}\right)(x) .
$$

Here we assumed that the compressible fluid is polytropic with the adiabatic exponent $\gamma$, and $\rho, v, S$, and $p$ denote the density, velocity, specific entropy, and pressure of a gas, respectively.

In hyperbolic conservation laws community, the formation of shocks has been studied extensively in the one-dimensional case $[7,8,12]$ using the method of characteristics, whereas for the multi-dimensional case the formation of singularities was investigated via indirect nonlinear functionals (e.g. total inertia) involving the hydrodynamics variables in $[16,20,21]$.

Next, we discuss Makino-Ukai-Kawashima's approach [16] on the break-down of the smooth solutions to the system (1.1) with a compactly supported initial data. In

\footnotetext{
*Received: March 15, 2009; accepted (in revised version): May 6, 2009. Communicated by Shi Jin.

$\dagger$ Department of Mathematics Sungkyunkwan University, Suwon 440-746, Korea (chae@skku.edu).

${ }^{\ddagger}$ Department of Mathematical Sciences, Seoul National University, Seoul 151-747, Korea (syha@snu.ac.kr).
} 
[16], the authors introduced the functional $H$ representing the total inertia:

$$
H(t):=\frac{1}{2} \int_{\mathbb{R}^{d}}|x|^{2} \rho(x, t) d x .
$$

Then they showed two ingredients on their blow-up analysis (uniform support of density and the uniform lower bound of $H^{\prime \prime}$ ) under the condition that there exist global smooth solutions:

$$
\exists R>0 \text { and } \beta=\min \left\{2, \frac{3}{\gamma-1}\right\} \text { independent of } t \text { satisfying }
$$

$$
\rho(x, t)=0, \quad|x| \geq R, \quad \text { and } \quad H^{\prime \prime}(t) \geq \beta E(t)=\beta E(0), \quad t \geq 0,
$$

where $E=E(t)$ is a total energy defined by

$$
E(t):=\int_{\mathbb{R}^{d}}\left[\frac{1}{2} \rho|v|^{2}+\frac{1}{\gamma-1} p\right] d x .
$$

Note that the functional $H$ satisfies

$$
H(t) \geq H(0)+H^{\prime}(0) t+\frac{\beta E(0)}{2} t^{2} .
$$

Since $H$ is uniformly bounded by a positive constant independent of $t$, more precisely

$$
H(t) \leq \frac{R^{2}}{2}\left\|\rho_{0}\right\|_{L^{1}},
$$

we obtain the contradiction in (1.3) as $t \rightarrow \infty$. This gives the finite time break-down of smooth solutions with a compactly supported initial data. Unfortunately, this global and indirect approach does not give us detailed information on the type of singularities and a blow-up rate, etc.

In this paper, we instead provide a local blow-up analysis which enables us to find the explicit blow-up dynamics of smooth solutions to the system (1.1) with a restricted class of initial data containing vacuum states. We use the method of characteristics as in one-dimensional case. As in [13], a vacuum state denotes any portion of $x-t$ plane in which $\rho=0$. We also distinguish two types of vacuum states (compression vacuum and rarefaction vacuums). In section 3 , we will show that shock waves should form in finite time at compression vacuum states.

For a given initial data (1.2), we define a singular set $\mathcal{S}$ leading to the finite-time blow-up of the divergence of fluid velocity:

$$
\mathcal{S}\left[\rho_{0}, v_{0}, S_{0}\right]:=\left\{a \in \mathbb{R}^{d} \mid \text { the point } a \text { satisfies the constraints }(I) \text { and }(I I)\right\} ;
$$

(I) The initial fluid velocity $v_{0}$ is irrotational and compressive at $a$ :

$$
\Omega_{0}(a)=0, \quad \operatorname{div}\left(v_{0}\right)(a):=\sum_{i=1}^{d} \partial_{i} v_{0}^{i}(a)<0, \quad \partial_{i}:=\frac{\partial}{\partial x_{i}} .
$$

Here $\Omega_{0}$ is the anti-symmetric part of the velocity gradient $\nabla v_{0}$ (see section $2)$. 
(II) The initial data is in $C^{3}(\Omega)$ and the initial density and entropy satisfy vacuum and boundedness conditions at $a$ :

$$
\sum_{|\alpha| \leq 2}\left|\partial^{\alpha} \rho_{0}(a)\right|=0, \quad \sum_{|\alpha| \leq 2}\left|\partial^{\alpha} S_{0}(a)\right|<\infty
$$

Here $\partial^{\alpha}=\partial_{1}^{\alpha_{1}} \cdots \partial_{d}^{\alpha_{d}}, \quad \alpha=\left(\alpha_{1}, \cdots, \alpha_{d}\right):$ multi-index in $\mathbb{Z}^{d}$.

The main theorem of this paper is as follows.

THEOREM 1.1. Suppose the singular set $\mathcal{S}$ corresponding to smooth initial data $\left(\rho_{0}, v_{0}, S_{0}\right)$ is nonempty, and let $(\rho, v, S)$ be the local smooth $C^{3}$-regular solutions in $\mathbb{R}^{d} \times\left[0, T_{*}\right)$ to the system (1.1)-(1.2) with $\gamma \in\{2\} \cup[3, \infty)$. Then the life-span $T_{*}$ of $C^{3}$-solutions is finite, and moreover it satisfies

$$
T_{*} \leq \inf _{a \in \mathcal{S}} \frac{d}{\left|\operatorname{div}\left(v_{0}\right)(a)\right|}
$$

REMARK 1.1. 1. The local existence theory for the classical smooth solutions to the system (1.1) with non-vacuum initial data was obtained in $[6,18]$. In contrast, for compactly supported initial data, the local existence of smooth solutions is also established in $[5,16]$.

2. The above estimate on the life-span of smooth solution does not depend on the specific domain. Hence the same result holds for a bounded domain with suitable boundary conditions.

3. In section 3 , we will show that the scalar quantity $\operatorname{div}(v):=\sum_{i=1}^{d} \partial_{i} v^{i}$ satisfies the scalar differential inequality along the particle trajectory issued from $a \in \mathcal{S}$ :

$$
\frac{D}{D t} \operatorname{div}(v) \leq-\frac{1}{d}(\operatorname{div}(v))^{2}, \quad \operatorname{div}\left(v_{0}\right)(a)<0
$$

Hence $|\operatorname{div}(v)|$ will blow up along the particle trajectories issued from the compression vacuum states in $\mathcal{S}$.

4. Note that in our setting with $d=1$, the sound speed $c$ satisfies

$$
\left.\frac{d c^{2}}{d x}\right|_{x=a}=\kappa \gamma(\gamma-1) e^{S} \rho^{\gamma-2}(a) \frac{d \rho}{d x}(a)=0, \quad \text { if } \quad a \in \mathcal{S}, \quad \gamma \geq 2,
$$

where $c=\sqrt{\partial_{\rho} p}$ is the speed of sound of the gas (see Remark 2.1). Hence our setting for the vacuum boundary problem is completely different from the physical vacuum singularity problem considered in $[12,14,23]$, where the speed of sound $c$ satisfies

$$
0<\left|\frac{d c^{2}}{d x}\right|<\infty
$$

2. Basic a priori estimates In this section, we study the time-evolution of hydrodynamic quantities along the particle trajectories. These estimates will be employed in the blow-up analysis in next section. 
The Euler equations (1.1) can be rewritten as a quasi-linear hyperbolic system for smooth solutions:

$$
\begin{aligned}
& \partial_{t} \rho+(v \cdot \nabla) \rho=-\rho \operatorname{div}(v), \quad x \in \mathbb{R}^{d}, \quad t>0, \\
& \partial_{t} v+(v \cdot \nabla) v=-\rho^{-1} \nabla p, \\
& \partial_{t} S+(v \cdot \nabla) S=0, \\
& p=\kappa e^{S} \rho^{\gamma}, \quad(\kappa>0, \quad \gamma>1) .
\end{aligned}
$$

Note that the term $\rho^{-1} \nabla p$ is well-defined even for vacuum states $\rho=0$ due to the constitutive relation for $p=\kappa e^{S} \rho^{\gamma}, \gamma>1$.

We next derive the evolution equations for the symmetric and anti-symmetric parts of the velocity gradient matrix $V:=\nabla v$. We use the second equation in (2.1) to find the Ricatti matrix equation:

$$
\partial_{t} V+(v \cdot \nabla) V=-V^{2}-\nabla\left(\rho^{-1} \nabla p\right) .
$$

We now decompose the velocity gradient $V$ and $\nabla\left(\rho^{-1} \nabla p\right)$ as a sum of a symmetric part and an anti-symmetric part respectively:

$$
\begin{aligned}
V & =\mathcal{D}+\Omega, \quad \mathcal{D}:=\frac{1}{2}\left[V+V^{T}\right], \quad \Omega:=\frac{1}{2}\left[V-V^{T}\right], \\
\nabla\left(\rho^{-1} \nabla p\right) & =\left(\nabla\left(\rho^{-1} \nabla p\right)\right)^{+}+\left(\nabla\left(\rho^{-1} \nabla p\right)\right)^{-}, \\
\left(\nabla\left(\rho^{-1} \nabla p\right)\right)^{ \pm} & =\frac{1}{2}\left[\nabla\left(\rho^{-1} \nabla p\right) \pm\left(\nabla\left(\rho^{-1} \nabla p\right)\right)^{T}\right] .
\end{aligned}
$$

Recall that $\mathcal{D}$ and $\Omega$ denote the deformation and vorticity tensors of the fluid (see $[1])$. Then $\mathcal{D}$ and $\Omega$ satisfy

$$
\begin{aligned}
& \frac{D}{D t} \mathcal{D}=-\mathcal{D}^{2}-\Omega^{2}-\left(\nabla\left(\rho^{-1} \nabla p\right)\right)^{+} \\
& \frac{D}{D t} \Omega=-\mathcal{D} \Omega-\Omega \mathcal{D}+\left(\nabla\left(\rho^{-1} \nabla p\right)\right)^{-} .
\end{aligned}
$$

In the following two lemmas, we estimate hydrodynamic quantities $\rho, S, \Omega$ and $\nabla\left(\rho^{-1} \nabla p\right)$ along the particle trajectories.

LEMma 2.1. Let $(\rho, v, S)$ be a smooth $C^{3}$-solutions in $\mathbb{R}^{d} \times\left[0, T_{*}\right)$ to the system $(2.1)$ corresponding to initial data $\left(\rho_{0}, v_{0}, S_{0}\right)$, and let $X=X(a, t)$ be the particle trajectory issued from $a \in \mathbb{R}^{d}$ at time $t=0$. Then we have

(i) $\sum_{|\alpha| \leq 2}\left|\partial^{\alpha} \rho(X(a, t), t)\right| \leq \sum_{|\alpha| \leq 2}\left|\partial^{\alpha} \rho_{0}(a)\right| \exp \left[C \int_{0}^{t}\left(\sum_{1 \leq|\alpha| \leq 3}\left|\partial^{\alpha} v(X(a, s), s)\right|\right) d s\right]$.

(ii) $\sum_{|\alpha| \leq 2}\left|\partial^{\alpha} S(X(a, t), t)\right| \leq \sum_{|\alpha| \leq 2}\left|\partial^{\alpha} S_{0}(a)\right| \exp \left[C \int_{0}^{t}\left(\sum_{1 \leq|\alpha| \leq 2}\left|\partial^{\alpha} v(X(a, s), s)\right|\right) d s\right]$.

Here $C$ is a positive constant independent of $t$.

Proof. (i) We differentiate the continuity equation

$$
\frac{D \rho}{D t}:=\partial_{t} \rho+(v \cdot \nabla) \rho=-\rho \operatorname{div} v,
$$


up to twice to find

$$
\begin{aligned}
& \frac{D}{D t} \partial_{j} \rho=-\partial_{j} v \cdot \nabla \rho-\partial_{j} \rho \operatorname{div} v-\rho \operatorname{div}\left(\partial_{j} v\right), \quad j=1, \cdots, d \\
& \begin{aligned}
\frac{D}{D t} \partial_{k} \partial_{j} \rho=- & \left(\partial_{j} \partial_{k} v \cdot \nabla\right) \rho-\left(\partial_{j} v \cdot \nabla\right) \partial_{k} \rho-\left(\partial_{k} v \cdot \nabla\right) \partial_{j} \rho \\
& \quad \partial_{j} \partial_{k} \rho \operatorname{div} v-\partial_{j} \rho \operatorname{div} \partial_{k} v-\rho \operatorname{div}\left(\partial_{j} \partial_{k} v\right)-\partial_{k} \rho \operatorname{div} \partial_{j} v, \quad j, k=1, \cdots, d .
\end{aligned}
\end{aligned}
$$

Then (2.4) and (2.5) imply

$$
\frac{D}{D t} \sum_{|\alpha| \leq 2}\left|\partial^{\alpha} \rho\right| \leq C\left[\sum_{1 \leq|\alpha| \leq 3}\left|\partial^{\alpha} v\right|\right] \sum_{|\alpha| \leq 2}\left|\partial^{\alpha} \rho\right| .
$$

We now integrate the above inequality (2.6) along the particle trajectory $X(a, t)$ to get the desired estimate.

(ii) By direct calculations, we have

$$
\begin{aligned}
& \frac{D S}{D t}=0, \quad \frac{D}{D t} \partial_{i} S=-\partial_{i} v \cdot \nabla S \\
& \frac{D}{D t} \partial_{j} \partial_{i} S=-\left[\left(\partial_{j} v\right) \cdot \nabla\right] \partial_{i} S-\left[\left(\partial_{j} \partial_{i} v\right) \cdot \nabla\right] S-\left[\partial_{i} v \cdot \nabla\right] \partial_{j} S .
\end{aligned}
$$

The equations (2.7) imply

$$
\frac{D}{D t} \sum_{|\alpha| \leq 2}\left|\partial^{\alpha} S\right| \leq C\left[\sum_{1 \leq|\alpha| \leq 2}\left|\partial^{\alpha} v\right|\right] \sum_{|\alpha| \leq 2}\left|\partial^{\alpha} S\right| .
$$

We integrate the above inequality along the particle trajectory to get the desired result. $\square$

REMARK 2.1. Note that under the assumption (II) on the initial mass density in section 1 , the mass density and its derivatives up to second order vanishes at $a \in \mathcal{S}$ along the smooth flow, i.e.,

$$
\sum_{|\alpha| \leq 2}\left|\partial^{\alpha} \rho_{0}(a)\right|=0 \quad \Longrightarrow \quad \sum_{|\alpha| \leq 2}\left|\partial^{\alpha} \rho(X(a, t), t)\right|=0 .
$$

Lemma 2.2. Let $(\rho, v, S)$ be a smooth $C^{3}$-solutions in $\mathbb{R}^{d} \times\left[0, T_{*}\right)$ to the system $(2.1)$ corresponding to initial data $\left(\rho_{0}, v_{0}, S_{0}\right)$, and let $X=X(a, t)$ be the particle trajectory mapping issued from $a \in \mathbb{R}^{d}$ at time $t=0$. Then we have

(i) $|\Omega(X(a, t), t)|$

$$
\begin{aligned}
& \leq\left|\Omega_{0}(a)\right| \exp \left[2 \int_{0}^{t}|D v(X(a, s), s)| d s\right] \\
& +\int_{0}^{t}\left|\left(\nabla\left(\rho^{-1} \nabla p\right)\right)^{-}(X(a, s), s)\right| \exp \left[2 \int_{s}^{t}|D v(X(a, \tau), \tau)| d \tau\right] d s .
\end{aligned}
$$

(ii) $\partial_{j}\left(\rho^{-1} \partial_{i} p\right)$

$$
=\kappa e^{S}\left[\left(\left(\partial_{i} S\right)\left(\partial_{j} S\right)+\partial_{j} \partial_{i} S\right) \rho^{\gamma-1}\right.
$$

$$
\left.+\left((\gamma-1)\left(\partial_{i} S\right)\left(\partial_{j} \rho\right)+\gamma\left(\partial_{j} S\right)\left(\partial_{i} \rho\right)+\gamma\left(\partial_{j} \partial_{i} \rho\right)\right) \rho^{\gamma-2}+\gamma(\gamma-2)\left(\partial_{j} \rho\right)\left(\partial_{i} \rho\right) \rho^{\gamma-3}\right] .
$$


Proof. (i) Note that the anti-symmetric part $\Omega$ of the velocity gradient $\nabla v$ satisfies

$$
\frac{D}{D t} \Omega=-\mathcal{D} \Omega-\Omega \mathcal{D}++\left(\nabla\left(\rho^{-1} \nabla p\right)\right)^{-} .
$$

This yields

$$
\frac{D}{D t}|\Omega| \leq 2|\mathcal{D}||\Omega|+\left|\left(\nabla\left(\rho^{-1} \nabla p\right)\right)^{-}\right|,
$$

where $|M|:=\sqrt{\sum_{i, j=1}^{d}\left|M_{i j}\right|^{2}}$, for any $d \times d$-matrix $M$. Hence Gronwall's lemma yields the desired result.

(ii) We use the constitute relation for $p=A e^{S} \rho^{\gamma}$ to find the desired result.

REMARK 2.2. For the case $\gamma \in\{2\} \cup[3, \infty)$, the right hand side of $\partial_{j}\left(\rho^{-1} \partial_{i} p\right)$ is bounded as long as $\sum_{|\alpha| \leq 2}\left|\partial^{\alpha} \rho\right|$ and $\sum_{|\alpha| \leq 2}\left|\partial^{\alpha} S\right|$ are bounded.

3. The proof of Theorem 1.1 In this section, we study the time-evolution of the scalar quantity $\operatorname{div}(v)$ as in [2], and show that this quantity will blow up along the particle trajectories issued from points in $\mathcal{S}$. This approach is a multi-dimensional counterpart of the method employed in the blow-up analysis of the one-dimensional inviscid Burgers's equation (see [8]).

Let $a \in \mathcal{S}$ be a singular point, i.e.,

$$
\Omega_{0}(a)=0, \quad \operatorname{div}\left(v_{0}\right)(a)<0, \quad \sum_{|\alpha| \leq 2}\left|\partial^{\alpha} \rho_{0}(a)\right|=0, \quad \sum_{|\alpha| \leq 2}\left|\partial^{\alpha} S_{0}(a)\right|<\infty .
$$

Consider a particle trajectory issued from $a \in \mathcal{S}$. Then it follows from Lemmas 2.1 and 2.2 that

$$
\begin{aligned}
& \sum_{|\alpha| \leq 2}\left|\partial^{\alpha} \rho(X(a, t), t)\right|=0, \quad \sum_{|\alpha| \leq 2}\left|\partial^{\alpha} S(X(a, t), t)\right|<\infty, \quad t \in\left[0, T_{*}\right), \\
& \Omega(X(a, t), t)=0, \quad\left(\nabla\left(\rho^{-1} \nabla p\right)\right)^{ \pm}(X(a, t), t)=0 .
\end{aligned}
$$

Then the equation for $\mathcal{D}$ becomes

$$
\frac{D}{D t} \mathcal{D}=-\mathcal{D}^{2}, \quad \text { along the particle trajectory } \quad X=X(a, t)
$$

Let $\lambda_{i},(i=1, \cdots, d)$ be the eigenvalues of $\mathcal{D}$. We now take the trace on both sides of the equation $(3.1)$, and use the relation $\operatorname{Tr}[\mathcal{D}]=\operatorname{div}(v)$ and an inequality

$$
\operatorname{Tr}\left[\mathcal{D}^{2}\right]=\sum_{i=1}^{d} \lambda_{i}^{2} \geq \frac{1}{d}\left(\sum_{i=1}^{d} \lambda_{i}\right)^{2}=\frac{1}{d}(\operatorname{div}(v))^{2},
$$

to find

$$
\frac{D}{D t}(\operatorname{div}(v)) \leq-\frac{1}{d}(\operatorname{div}(v))^{2}
$$

Hence we have an explicit upper bound for $\operatorname{div}(v)$ along the particle trajectory:

$$
\operatorname{div}(v)(X(a, t), t) \leq \frac{d \operatorname{div}\left(v_{0}\right)(a)}{d+\left(\operatorname{div}\left(v_{0}\right)(a)\right) t} .
$$


Since $\operatorname{div} v_{0}(a)<0, \operatorname{div} v$ will blow up before the critical time $t=t_{c}(a)$ :

$$
t_{c}(a):=-\frac{d}{\operatorname{div}\left(v_{0}\right)(a)} .
$$

Therefore the life-span $T_{*}$ should satisfy

$$
T_{*} \leq-\frac{d}{\operatorname{div}\left(v_{0}\right)(a)}, \quad a \in \mathcal{S} .
$$

This completes the proof of Theorem 1.1.

REMARK 3.1. In [17], page 182, the authors stated the following sentences for the possible phenomena on the boundary of support "Probably a shock appear on the boundary of the support. However the verification is a subject of the future study" . Apparently our result in Theorem 1.1 can answer the shock formation at the point $a \in \mathcal{S} \cap$ boundary of support for the Euler equations with $\gamma \in\{2\} \cup[3, \infty)$.

Acknowledgment. Both authors would like to appreciate Y. Brenier, T.-P. Liu, and Y. Sone for their stimulating discussions. The research of D. Chae was supported partially by KRF Grant(MOEHRD, Basic Research Promotion Fund), and the work of S.-Y. Ha is partially supported by KRF-2008-C00023.

\section{REFERENCES}

[1] A. Bertozzi and A. Majda, Vorticity and Incompressible Flow. Cambridge Texts in Applied Mathematics 27, Cambridge University Press. 2002.

[2] D. Chae and E. Tadmor, On the finite time blow-up of the Euler-Poisson equations in $\mathbb{R}^{N}$. Commun. Math. Sci. 6, 785-789, 2008.

[3] D. Christodoulou, The Euler equations of compressible fluid flow. Bull. Amer. Math. Soc. (N.S.) 44, 581-602, 2007.

[4] Y. Guo and S. Tahvildar-Zadeh, Formation of singularities in relativistic fluid dynamics and in spherically symmetric plasma physics. Contemp. Math. 238, 151-161, 1999.

5] J. Jang and N. Masmoudi, Well-posedness for compressible Euler equations with physical vacuum singularity. Preprint.

[6] T. Kato, The Cauchy problem for quasi-linear symmetric hyperbolic systems. Arch. Ration. Mech. Anal. 58, 181-205, 1975.

[7] P. Lax, Development of singularity of solutions of nonlinear hyperbolic partial differential equations. J. Math. Phys. 5, 611-613, 1964.

[8] P. Lax, The formation and decay of shock waves. Amer. Math. Monthly 79, 227-241, 1972.

[9] H.-L. Li, J. Li and Z. Xin, Vanishing of vacuum states and blow-up phenomena of the compressible Navier-Stokes equations. Commun. Math. Phys. 281, 401-444, 2008.

[10] L. W. Lin, On the vacuum state for the equations of isentropic gas dynamics. J. Math. Anal. Appl. 121, 406-425, 1987.

[11] L. W. Lin and X. P. Ye, Conjecture on the non-appearance of vacuum states in isentropic gas flow with shocks. Proceedings of the fifth international colloquium differential equations. Provdiv 1994.

[12] T.-P. Liu, The development of singularity in the nonlinear waves for quasi-linear hyperbolic partial differential equations. J. Differential Equations 33, 92-111, 1979.

[13] T.-P. Liu and J. A. Smoller, On the vacuum state for the isentropic gas dynamics equations. Adv. in Appl. Math. 1, 345-359, 1980.

[14] T.-P. Liu and T. Yang, Compressible flow with vacuum and physical singularity. Methods and Appl. Anal. 7, 495-510, 2000.

[15] T. Luo, Z. Xin and T. Yang, Interface behavior of compressible Navier-Stokes equations with vacuum. SIAM. J. Math. Anal. 31, 1175-1191, 2000.

[16] T. Makino, S. Ukai and S. Kawashima, Sur la solutions á support compact de l's equation d's Euler compressible. Japan. J. Appl. Math. 3, 249-257, 1986. 
[17] T. Makino, S. Ukai and S. Kawashima, On compactly supported solutions of the compressible Euler equation. Recent Topics in Nonlinear PDE III, 173-183, North-Holland Math. Stud. 148, Noth-Holland, Amsterdam, 1987.

[18] A. Majda, Compressible Fluid Flow and Systems of Conservation Laws in Several Space Variables. Applied Mathematical Sciences 53, Springer-Verlag, New York, 1984.

[19] R. Pan and J. A. Smoller, Blowup of smooth solutions for relativistic Euler equations. Commun. Math. Phys. 262, 729-755, 2006.

[20] T. Sideris, Formation of singularity in three-dimensional compressible fluids. Commun. Math. Phys. 101, 475-485, 1984.

[21] T. Sideris, Formation of singularity of solutions to nonlinear hyperbolic equations. Arch. Rational Mech. Anal. 86, 369-381, 1984.

[22] Z. Xin, Blowup of smooth solutions to the compressible Navier-Stokes equation with compact density. Commun. Pure Appl. Math. 51, 229-240, 1998.

[23] T. Yang, Singular behavior of vacuum states for compressible fluids. J. Comput. Appl. Math. 190, 211-231, 2006. 